Original Research Paper

\title{
Phenotypic Evaluation of Heritability, Agro-Morphological and Yield Characters of Sixteen Amaranthus Linn. Genotypes
}

\author{
Onuoha Samuel Ogechi and Olawuyi Odunayo Joseph \\ Genetics and Molecular Biology Unit, Department of Botany, University of Ibadan, Ibadan, Nigeria
}

\author{
Article history \\ Received: $17-02-2017$ \\ Revised: $10-04-2017$ \\ Accepted: 20-07-2017 \\ Corresponding Author: \\ Onuoha Samuel Ogechi \\ Genetics and Molecular \\ Biology Unit, Department of \\ Botany, University of Ibadan, \\ Ibadan, Nigeria \\ Email: sam4christ2005@gmail.com
}

\begin{abstract}
The field experiment was conducted to evaluate the heritability, genetic variance, agro-morphological and yield characters of Sixteen Amaranthus genotypes. The seeds of the sixteen (16) genotypes of Amaranthus evaluated in this study were; NG/AA/MAY/09/027, NG/AA/03/11/010, NG/AO/11/08/042, NG/AO/11/08/039, NG/SA/DEC/07/0423， NG/SA/DEC/07/0412， NGB01667， NGB01601, NGB01283, NGB01271, NGB01276, NGB01259, NGB01644, NGB01234, NGB01613 and NGB01662. The results showed that there were variability performances in growth and yield characters of Amaranthus genotypes. NG/AA/MAY/09/027 and NG/AO/11/08/039 had the best growth characters while $\mathrm{NG} / \mathrm{AO} / 11 / 08 / 042$ had best yield performance compared to other genotypes. The stem length recorded the best heritability estimate of $95.5 \%$ while weight of dry leaf, weight of fresh and dry inflorescent had least $(47.7 \%)$. The plant height had a positive significant correlation with number of leaf $(r=0.53)$, leaf width $(r=0.57)$, number of branches $(r=$ $0.56)$ but a strong positive correlation with stem length $(\mathrm{r}=0.97)$, stem girth $(r=0.75)$, number of inflorescent $(r=0.68)$, inflorescent length $(0.64)$ and inflorescent width $(r=0.72)$. Prin. 1 accounted for the highest variation in growth and yield characters with proportion of 0.3376 and eigen value of 4.7269, while Prin. 14 was the least with proportion of 0.0003 and eigen value of 0.0038 . Therefore, there could be genetic improvement of $\mathrm{NG} / \mathrm{AA} / \mathrm{MAY} / 09 / 027$ and NG/AO/11/08/039 genotypes for further improvement of Amaranthus.
\end{abstract}

Keywords: Amaranthus, Heritability, Agro-Morphological, Variance

\section{Introduction}

The genus Amaranthus of the order Caryophillalales comprises of more than 60 species $\mathrm{C} 4$ dicotyledonous herbaceous plants. Amaranthus species are cultivated in Central and South America, Africa and some parts of Asia as ornamentals, some are a source of highly nutritious pseudo-cereals and vegetables while others are notoriously weeds (Holm et al., 1997; Steckel, 2007). It has received attention due to its essential nutrients for the human diet (Tucker, 1986; Bressani et al., 1992). Amaranthus species are tolerant to infestation by herbivorous insects under field conditions and can grow successfully under varied soil and agro-climatic conditions such as bright sunlight, high temperatures and low moisture (Prakash and Pal, 1991; Brenner et al., 2010; Angel and Paulina, 2011). It can also tolerate a variety of unfavorable soil conditions such as high salinity, acidity, or alkalinity (Tucker, 1986). Besides other crops, cultivation of this vegetable will not only increase food production but also provide balanced nutrition, food security, health security and poverty alleviation (Buragohain et al., 2013).

Despite the perceived usefulness and untapped potentials of this vegetable, Amaranthus are underutilized making their potential economic value remaining "underexploited". It has also been reported that it has been neglected for many years by researchers, policy makers and funding agencies and thus currently threatened by extinction. Hence, improvement of this vegetable is highly needed to ensure maximum agronomic yield and high productivity of Amaranthus with a view to conserve the germplasm.

This study aimed at evaluating the heritability, genetic variance, agro-morphological and yield characters of Sixteen Amaranthus genotypes so as to improve the production of Amaranthus spp. for proper documentation of Amaranthus germplasm. 


\section{Materials and Method}

\section{Experimental Site and Amaranthus Germplasm}

This study was carried out at the Nursery Farm of the Department of Botany, University of Ibadan, Oyo state, Nigeria. The site lies between Latitude $7^{\circ} 02^{\prime} 49^{\prime \prime}$ and $7^{\circ} 43^{\prime} 21^{\prime \prime} \mathrm{N}$ longitude $3^{\circ} 31^{\prime} 58^{\prime \prime}$ and $4^{\circ} 08^{\prime} 20^{\prime \prime} \mathrm{E}$ with an altitude of $150 \mathrm{~m}$ in the valley at $275 \mathrm{~m}$ above sea level at moderate annual rainfall of 1,205 $\mathrm{mm}$ (Amanambu and Egbinola, 2013). The seeds of the sixteen Amaranthus spp. Genotypes were sourced from the National Centre for Genetic Resources and Biotechnology (NACGRAB), Moor plantation, Ibadan, Nigeria. The genotypes were NG/AA/MAY/09/027, NG/AA/03/11/010,

$\mathrm{NG} / \mathrm{AO} / 11 / 08 / 039$,

$\mathrm{NG} / \mathrm{AO} / 11 / 08 / 042$,

NG/SA/DEC/07/0412, NGB01667, NGB01601, NGB01283, NGB01271, NGB01276, NGB01259, NGB01644, NGB01234, NGB01613 and NGB01662.

\section{Experimental Design, Plant Spacing and Planting Method}

The Experiment was a complete Randomized Design with the polythene bags properly spaced at a distance of $65 \mathrm{~cm}$ between genotypes and $45 \mathrm{~cm}$ within genotypes. The young shoots were transplanted in pairs in each labeled polythene bags replicated four times. The seeds were first planted in the nursery through broadcasting for three weeks before transplanting in pairs to the well-labeled experimental polythene bags at the Nursery Farm of the Department of Botany, University of Ibadan, Oyo state, Nigeria. The cultivation was monitored and watered daily to resist drought.

\section{Data Collection}

After one week of transplanting, data taken weekly on growth characters included plant height $(\mathrm{cm})$, number of leaves, stem length $(\mathrm{cm})$, stem girth $(\mathrm{cm})$ and leaf area $\left(\mathrm{cm}^{2}\right)$. The plant height, stem length and leaf area were measured using a meter rule while stem girth was measured with a vernier caliper. Quantitative and qualitative data on flower characters were collected at maturity for six (6) weeks, this included number of inflorescence, inflorescence width $(\mathrm{cm})$, inflorescence length $(\mathrm{cm})$ and number of branches. The number of inflorescence and number of branches were done by counting, while inflorescence width and inflorescence length were determined by measurement using a metre rule. The inflorescence color and plant color were also determined by observation. After harvesting, the biomass of fresh and dry inflorescence and leaves were determined for each of the genotype using weighing balance. Heritability and genetic variance was also determined using these formulas:

$$
\text { Genotypic variance }=\frac{\text { Genotype } M S-\text { Error } M S}{\text { Replicate }}
$$

Phenotypic Variance: Genotypic Variance + Error MS

$$
\text { Heritability }=\frac{\text { Genotypic Variance }}{\text { Phenotypic Variance }}
$$

\section{Statistical Analysis}

The data were subjected to Analysis of Variance (ANOVA) and difference in means was separated using DMRT at $95 \%$ probability level $(\mathrm{p}<0.05)$. The relationship among the quantitative and qualitative traits were established using Pearson correlation coefficient and Principal Component Analysis (PCA). In addition, Heritability, Phenotypic Coefficient of Variance (PCV), Genotypic Coefficient of Variance (GCV) were also determined.

\section{Results}

Qualitative Traits in Amaranthus Genotypes

The qualitative traits observed in genotypes of Amaranthus are shown in Table 1. Genotypes NG/AA/MAY/09/027, NG/AA/03/11/010, NG/AO/11/08/039, NGB01601, NGB01271, NGB01276, NGB01259, NGB01644, NGB01234 and NGB01662 had a plant and spike/inflorescence color of green while $\mathrm{NG} / \mathrm{AO} / 11 / 08 / 042$, NG/SA/DEC/07/0423, NG/SA/DEC/07/0412, NGB01667, NGB01283 and NGB01613 had plant and spike/inflorescence color of green but with a shade of purple. The spike/inflorescence colors were observed to vary from green to green with a shade of purple. The grain colors were observed to be TAN which is a light-brown color across all the genotype.

\section{Mean Square Variance of Growth Characters in Amaranthus Genotypes}

The result of the mean square variance of growth character for Amaranthus from Table 2 shows that the genotype and weeks after planting produced significant $(p<0.01)$ effect on Plant height, Number of leaves, Stem length, Stem girth and Leaf width but non-significant on Leaf length for both genotype and week.

\section{Mean Square Variance of Yield Characters in Amaranthus Genotypes}

The result of the mean square variance of yield characters in Table 3 shows that the genotype and weeks after planting produced significant effect $(p<0.01)$ on Number of Inflorescence, Inflorescence length, Inflorescence width, Number of branches, Fresh leaf biomass, Weight of dry leaf, Weight of fresh inflorescent and Weight of dry inflorescent. 
Table 1. Qualitative traits in Amaranthus genotypes

\begin{tabular}{lllll}
\hline S/N & Genotypes & Plant color & Inflorescence colour & Grain color \\
\hline 1 & NG/AA/MAY/09/027 & Green & Green & TAN \\
2 & NG/AA/03/11/010 & Green & Green & TAN \\
3 & NG/AO/11/08/042 & Green with a shade of purple & Green with some purple & TAN \\
4 & NG/AO/11/08/039 & Green & Green & TAN \\
5 & NG/SA/DEC/07/0423 & Green with a shade of purple & Green & TAN \\
6 & NG/SA/DEC/07/0412 & Green with a shade of purple & Purple & TAN \\
7 & NGB01667 & Green with a shade of purple & Green with some purple & TAN \\
8 & NGB01601 & Green & Green & TAN \\
9 & NGB01283 & Green with a shade of purple & Green & TAN \\
10 & NGB01271 & Green & Green & TAN \\
11 & NGB01276 & Green & Green & TAN \\
12 & NGB01259 & Green & Green & TAN \\
13 & NGB01644 & Green & Green & TAN \\
14 & NGB01234 & Green & Green with some purple & TAN \\
15 & NGB01613 & Green with a shade of purple & Green & TAN \\
16 & NGB01662 & Green & & TAN \\
\hline
\end{tabular}

Table 2. Mean square variance of growth characters at different stages in Amaranthus genotypes

\begin{tabular}{llllllll}
\hline Source of variation & Df & Plant height & Number of leaves & Stem length & Stem girth & Leaf length & Leaf width \\
\hline Genotype & 15 & $5388.43^{* * *}$ & $9750.21^{* * *}$ & $3929.57^{* * *}$ & $0.69^{* * *}$ & $130.70^{\text {ns }}$ & $28.79^{* * *}$ \\
Weeks & 8 & $85011.33^{* * *}$ & $1480897^{* * *}$ & $50962.01^{* * *}$ & $2.53^{* * *}$ & $200.01^{\text {ns }}$ & $43.57^{* * *}$ \\
Replicates & 3 & 3.97 & 4.14 & 2.67 & 0.03 & 1.08 & 0.17 \\
Model & 26 & 29266.49 & 10182.21 & 17947.99 & 1.18 & 137.07 & 30.04 \\
Error & 549 & 100.28 & 267.14 & 46.09 & 0.02 & 3.18 & 0.60 \\
Corrected total & 575 & & & & \\
$*$
\end{tabular}

Table 3. Mean square variance of yield characters at different stages in Amaranthus genotypes

\begin{tabular}{|c|c|c|c|c|c|c|c|c|c|}
\hline $\begin{array}{l}\text { Source of } \\
\text { Variation }\end{array}$ & Df & $\begin{array}{l}\text { Number of } \\
\text { Inflorescence }\end{array}$ & $\begin{array}{l}\text { Inflorescence } \\
\text { length }\end{array}$ & $\begin{array}{l}\text { Inflorescence } \\
\text { width }\end{array}$ & $\begin{array}{l}\text { Number } \\
\text { of branches }\end{array}$ & $\begin{array}{l}\text { Fresh leaf } \\
\text { biomass }\end{array}$ & $\begin{array}{l}\text { Weight of } \\
\text { dry leaf } \\
\text { biomass }\end{array}$ & $\begin{array}{l}\text { Weight of } \\
\text { fresh } \\
\text { inflorescent }\end{array}$ & $\begin{array}{l}\text { Weight of } \\
\text { dry } \\
\text { inflorescent }\end{array}$ \\
\hline Genotype & 15 & $676.76^{* * *}$ & $317.62 * * *$ & $31.28^{* * *}$ & $72.69 * * *$ & $4.37 * * *$ & $0.44 * * *$ & $55.02 * * *$ & $10.65^{* * *}$ \\
\hline Weeks & 8 & $13579.17 * * *$ & $12084.07 * * *$ & $718.93 * * *$ & $793.67 * * *$ & $614.38 * * *$ & $92.06^{* * *}$ & $6001.86 * * *$ & $1691.68^{* * *}$ \\
\hline Replicate & 3 & $216.58^{\mathrm{ns}}$ & $14.14^{\mathrm{ns}}$ & $6.21^{\mathrm{ns}}$ & $8.05^{\mathrm{ns}}$ & $0.00^{\mathrm{ns}}$ & $0.00^{\mathrm{ns}}$ & $0.00^{\mathrm{ns}}$ & $0.00^{\mathrm{ns}}$ \\
\hline Model & 26 & 4593.63 & 3903.05 & 239.97 & 287.07 & 191.56 & 28.58 & 1878.47 & 526.66 \\
\hline Error & 549 & 116.32 & 44.87 & 4.52 & 5.95 & 0.96 & 0.09 & 12.03 & \\
\hline Corrected total & 575 & & & & & & & & \\
\hline
\end{tabular}

\section{Genotypic Effect of Growth Characters in Amaranthus Genotypes}

The result of the mean performance of genotypic effect on growth character of Amaranthus reveals significant $(\mathrm{p}<0.01)$ effect on Amaranthus genotypes as shown in Table 4. NG/AA/MAY/09/027 was significantly higher for Plant height and Stem length compared to other genotypes. Also, leaf width produced significant effect for NGB01271 while Stem girth and Leaf length were significantly higher for $\mathrm{NG} / \mathrm{AO} / 11 / 08 / 039$ but different from other genotypes. NGB01644 was significantly higher for Number of leaves than other genotypes.

\section{Genotypic Effect of Yield Characters in Amaranthus Genotypes}

The result in Table 5 shows that the genotypic effect of Amaranthus yield related character was significant at $\mathrm{P}<0.05$. NG/SA/DEC/07/0412 was significantly higher for Number of inflorescence and weight of fresh inflorescent yield compared to other genotypes. For Number of inflorescence NG/SA/DEC/07/0412 did not differ statistically from genotype $\mathrm{NG} / \mathrm{AO} / 11 / 08 / 042$, $\mathrm{NG} / \mathrm{AO} / 11 / 08 / 039$,

NG/AS/DEC/07/0423,

NG/AS/DEC/07/0412, NGB01601 and NGB01276. For weight of fresh inflorescent NG/SA/DEC/07/0412 did not differ statistically from genotypes NG/AO/11/08/039, NGB01667 and NGB01234. Also, 
Inflorescence length was higher for NG/AO/11/08/039 while Inflorescence width and Fresh leaf biomass were significantly higher for $\mathrm{NG} / \mathrm{AO} / 11 / 08 / 042$ but different from other genotypes. However, it did not differ statistically from genotypes NG/SA/DEC/07/0412 and NGB01601. For Inflorescence width, NG/AO/11/08/042 did not differ statistically from genotypes NG/AO/11/08/039, NG/SA/DEC/07/0423, NG/SA/DEC/07/0412, NGB01667 and NGB01283. For Fresh leaf biomass, NG/AO/11/08/042 did not differ statistically from genotypes NG/AA/MAY/09/027, NG/AO/11/08/039 and NGB01601. NGB01601 had higher Number of branches than other genotypes while NGB01667 is significant for weight of dry leaf biomass and weight of dry inflorescent. However, NGB01667 did not differ statistically from NG/AO/11/08/039, NG/SA/DEC/07/0423, NG/SA/DEC/07/0412, NGB01283, NGB01271 and NGB01662 for the weight of dry leaf biomass. Also, for the weight of dry inflorescent NGB01667did not differ statistically from genotypes NG/AO/11/08/039, NG/SA/DEC/07/0423, NG/SA/DEC/07/0412, NGB01283, NGB01271, NGB01234 and NGB01662.

\section{Heritability and Genotypic variance of Growth and Yield Traits of Amaranthus Genotype}

The result of the component of variance for growth and yield traits in Amaranthus shown in Table 6 reveals that the phenotypic variance of both growth and yield characters were higher than the genotypic variance in all the characters evaluated. The values for the phenotypic and genotypic variance were highest at Number of leaves but least at weight of dry leaf. The stem length recorded the best heritability estimate of $95.5 \%$ while weight of dry leaf, weight of fresh and dry inflorescent had least (47.7\%).

Table 4. Genotypic Effect of Growth Characters in Amaranthus genotypes

\begin{tabular}{|c|c|c|c|c|c|c|}
\hline Genotype & $\begin{array}{l}\text { Plant height } \\
(\mathrm{cm})\end{array}$ & $\begin{array}{l}\text { Number of } \\
\text { leaves }\end{array}$ & $\begin{array}{l}\text { Stem length } \\
\text { (cm) }\end{array}$ & $\begin{array}{l}\text { Stem girth } \\
\text { (cm) }\end{array}$ & $\begin{array}{l}\text { Leaf length } \\
\text { (cm) }\end{array}$ & $\begin{array}{l}\text { Leaf width } \\
(\mathrm{cm})\end{array}$ \\
\hline NG/AA/MAY/09/027 & $98.64^{\mathrm{a}}$ & $37.28^{\mathrm{efg}}$ & $87.31^{\mathrm{a}}$ & $1.21^{\mathrm{b}}$ & $15.74^{\mathrm{e}}$ & $7.49^{\mathrm{de}}$ \\
\hline $\mathrm{NG} / \mathrm{AA} / 03 / 11 / 010$ & $51.70^{\mathrm{f}}$ & $23.61^{\mathrm{i}}$ & $41.48^{\mathrm{i}}$ & $0.69^{\mathrm{i}}$ & $12.13^{\mathrm{g}}$ & $4.83^{\mathrm{j}}$ \\
\hline $\mathrm{NG} / \mathrm{AO} / 11 / 08 / 042$ & $88.59^{\mathrm{bc}}$ & $48.14^{\mathrm{d}}$ & $72.68^{\mathrm{cd}}$ & $1.11^{\mathrm{def}}$ & $16.36^{\mathrm{cde}}$ & $7.11^{\mathrm{fg}}$ \\
\hline $\mathrm{NG} / \mathrm{AO} / 11 / 08 / 039$ & $78.09^{\mathrm{d}}$ & $60.92^{\mathrm{bc}}$ & $68.77^{\text {ef }}$ & $1.33^{\mathrm{a}}$ & $21.03^{\mathrm{a}}$ & $7.89^{\mathrm{bc}}$ \\
\hline NG/SA/DEC/07/0423 & $85.12^{\mathrm{c}}$ & $32.28^{\mathrm{gh}}$ & $67.09^{\text {ef }}$ & $1.22^{\mathrm{b}}$ & $18.29^{\mathrm{b}}$ & $8.11^{\mathrm{b}}$ \\
\hline $\mathrm{NG} / \mathrm{SA} / \mathrm{DEC} / 07 / 0412$ & $91.69^{\mathrm{b}}$ & $44.86^{\mathrm{de}}$ & $78.09^{\mathrm{b}}$ & $1.08^{\text {efg }}$ & $18.12^{\mathrm{b}}$ & $7.57^{\text {cde }}$ \\
\hline NGB01667 & $85.45^{\mathrm{c}}$ & $29.33^{\text {ghi }}$ & $73.16^{\mathrm{c}}$ & $1.14^{\text {cde }}$ & $16.70^{\text {cde }}$ & $7.98^{\mathrm{b}}$ \\
\hline NGB01601 & $73.85^{\text {de }}$ & $42.00^{\operatorname{def}}$ & $62.49^{\mathrm{g}}$ & $1.11^{\mathrm{def}}$ & $17.29^{\mathrm{c}}$ & $7.84^{\text {bcd }}$ \\
\hline NGB01283 & $83.85^{\mathrm{c}}$ & $22.81^{\mathrm{i}}$ & $69.67^{\text {cde }}$ & $1.10 \mathrm{def}$ & $14.73^{\mathrm{f}}$ & $7.25^{\mathrm{ef}}$ \\
\hline NGB01271 & $85.29^{\mathrm{c}}$ & $27.89^{\mathrm{hi}}$ & $69.76^{\text {cde }}$ & $1.14^{\text {cde }}$ & $16.07^{\mathrm{de}}$ & $8.47^{\mathrm{a}}$ \\
\hline NGB01276 & $72.64^{\mathrm{e}}$ & $34.97^{\text {fgh }}$ & $62.63^{\mathrm{g}}$ & $1.02^{\mathrm{gh}}$ & $16.28^{\mathrm{de}}$ & $7.07^{\mathrm{fg}}$ \\
\hline NGB01259 & $72.93^{\mathrm{de}}$ & $56.56^{\mathrm{c}}$ & $62.28^{\mathrm{g}}$ & $1.07^{\mathrm{fg}}$ & $16.42^{\text {cde }}$ & $6.49^{\mathrm{hi}}$ \\
\hline NGB01644 & $77.94^{\mathrm{d}}$ & $80.83^{\mathrm{a}}$ & $69.25^{\mathrm{def}}$ & $1.19^{\mathrm{bc}}$ & $16.10^{\mathrm{de}}$ & $6.81^{\mathrm{gh}}$ \\
\hline NGB01234 & $78.03^{\mathrm{d}}$ & $66.33^{\mathrm{b}}$ & $69.99^{\text {cde }}$ & $1.17_{\text {cde }}$ & $16.68^{\text {cde }}$ & $6.71^{\mathrm{gh}}$ \\
\hline NGB01613 & $75.15^{\mathrm{de}}$ & $32.39^{\text {gh }}$ & $65.88^{\mathrm{f}}$ & $1.06^{\mathrm{fg}}$ & $16.74^{\mathrm{cd}}$ & $6.72^{\mathrm{gh}}$ \\
\hline NGB01662 & $54.41^{\mathrm{f}}$ & $37.11^{\text {efg }}$ & $50.09^{h}$ & $0.99^{\mathrm{h}}$ & $14.46^{\mathrm{f}}$ & $6.14^{\mathrm{i}}$ \\
\hline
\end{tabular}

Mean with the same letter in the same column are not significantly at $\mathrm{p} \geq 0.05$ according to Duncan Multiple Range Test (DMRT)

Table 5. Genotypic Effect of Yield Characters in Amaranthus genotypes

\begin{tabular}{|c|c|c|c|c|c|c|c|c|}
\hline Genotype & $\begin{array}{l}\text { Number of } \\
\text { Inflorescence }\end{array}$ & $\begin{array}{l}\text { Inflorescence } \\
\text { length }(\mathrm{cm})\end{array}$ & $\begin{array}{l}\text { Inflorescence } \\
\text { width }(\mathrm{cm})\end{array}$ & $\begin{array}{l}\text { Number } \\
\text { of branches }\end{array}$ & $\begin{array}{l}\text { Fresh leaf } \\
\text { biomass }(\mathrm{g})\end{array}$ & $\begin{array}{l}\text { Dry leaf } \\
\text { biomass }(\mathrm{g})\end{array}$ & $\begin{array}{l}\text { Weight of fresh } \\
\text { inflorescent }(\mathrm{g})\end{array}$ & $\begin{array}{l}\text { Weight of dry } \\
\text { inflorescent }(\mathrm{g})\end{array}$ \\
\hline$\overline{\mathrm{NG} / \mathrm{AA} / \mathrm{MAY} / 09 / 027}$ & $8.14^{\mathrm{d}}$ & $4.50^{\mathrm{e}}$ & $1.53^{\mathrm{g}}$ & $3.06^{\text {bcde }}$ & $1.38^{\mathrm{abc}}$ & $0.36^{\mathrm{de}}$ & $2.33^{\text {cde }}$ & $1.51^{\mathrm{bcd}}$ \\
\hline $\mathrm{NG} / \mathrm{AA} / 03 / 11 / 010$ & $9.19^{\mathrm{cd}}$ & $5.51^{\mathrm{e}}$ & $2.26^{\mathrm{efg}}$ & $1.64^{\mathrm{fg}}$ & $0.73^{\mathrm{d}}$ & $0.16^{\mathrm{f}}$ & $1.69^{\mathrm{e}}$ & $0.67^{\mathrm{e}}$ \\
\hline $\mathrm{NG} / \mathrm{AO} / 11 / 08 / 042$ & $20.31^{\mathrm{a}}$ & $11.24^{\mathrm{bc}}$ & $4.74^{\mathrm{a}}$ & $3.11^{\text {bcde }}$ & $1.83^{\mathrm{a}}$ & $0.37^{\mathrm{de}}$ & $3.83^{\text {bcd }}$ & $1.51^{\text {cde }}$ \\
\hline $\mathrm{NG} / \mathrm{AO} / 11 / 08 / 039$ & $20.69^{\mathrm{a}}$ & $15.61^{\mathrm{a}}$ & $4.14^{\mathrm{abc}}$ & $4.08^{\mathrm{ab}}$ & $1.56^{\mathrm{ab}}$ & $0.43^{\mathrm{abcd}}$ & $3.98^{\mathrm{abc}}$ & $1.81^{\mathrm{abcd}}$ \\
\hline NG/SA/DEC/07/0423 & $13.31^{\text {bcd }}$ & $9.76^{\mathrm{dc}}$ & $3.78^{\text {abcd }}$ & $1.06^{\mathrm{gh}}$ & $0.89^{\text {cd }}$ & $0.56^{\mathrm{ab}}$ & $3.29^{\text {cde }}$ & $2.52^{\mathrm{a}}$ \\
\hline NG/SA/DEC/07/0412 & $21.06^{\mathrm{a}}$ & $12.65^{\mathrm{abc}}$ & $4.43^{\mathrm{ab}}$ & $2.72^{\text {cdef }}$ & $0.87^{\text {cd }}$ & $0.43^{\mathrm{abcd}}$ & $5.76^{\mathrm{a}}$ & $2.15^{\mathrm{abc}}$ \\
\hline NGB01667 & $12.08^{\mathrm{bcd}}$ & $10.44^{\mathrm{c}}$ & $3.96^{\mathrm{abc}}$ & $0.39^{\mathrm{h}}$ & $0.65^{\mathrm{d}}$ & $0.58^{\mathrm{a}}$ & $4.00^{\mathrm{abc}}$ & $2.58^{\mathrm{a}}$ \\
\hline NGB01601 & $19.97^{\mathrm{a}}$ & $13.92^{\mathrm{ab}}$ & $3.19^{\text {cde }}$ & $5.00^{\mathrm{a}}$ & $1.37^{\mathrm{abc}}$ & $0.36^{\mathrm{de}}$ & $2.99^{\text {cde }}$ & $1.02^{\mathrm{de}}$ \\
\hline NGB01283 & $12.83^{\text {bcd }}$ & $11.35^{\mathrm{bc}}$ & $3.81^{\mathrm{abcd}}$ & $0.00^{\mathrm{h}}$ & $0.73^{\mathrm{d}}$ & $0.44^{\mathrm{abcd}}$ & $2.96^{\text {cde }}$ & $2.14^{\mathrm{abc}}$ \\
\hline NGB01271 & $14.00^{\text {bcd }}$ & $9.76^{\mathrm{dc}}$ & $3.41^{\text {bcd }}$ & $1.19^{\mathrm{gh}}$ & $1.02^{\mathrm{cd}}$ & $0.54^{\mathrm{abc}}$ & $2.61^{\text {cde }}$ & $2.31^{\mathrm{ab}}$ \\
\hline NGB01276 & $15.86^{\mathrm{ab}}$ & $10.25^{\mathrm{c}}$ & $3.14^{\text {cde }}$ & $2.50^{\mathrm{def}}$ & $1.13^{\mathrm{bcd}}$ & $0.24^{\mathrm{ef}}$ & $2.67^{\text {cde }}$ & $1.01^{\mathrm{de}}$ \\
\hline NGB01259 & $12.50^{\mathrm{bcd}}$ & $9.36 \mathrm{~d}^{\mathrm{c}}$ & $2.80^{\mathrm{def}}$ & $3.97^{\mathrm{abc}}$ & $1.11^{\text {bcd }}$ & $0.40^{\text {bcde }}$ & $2.07^{\mathrm{de}}$ & $1.66^{\text {bcd }}$ \\
\hline NGB01644 & $11.47^{\mathrm{bcd}}$ & $9.19 \mathrm{~d}^{\mathrm{c}}$ & $2.77^{\mathrm{def}}$ & $3.64^{\text {bcd }}$ & $0.61^{\mathrm{d}}$ & $0.34^{\mathrm{de}}$ & $4.19^{\mathrm{abc}}$ & $1.63^{\text {bcd }}$ \\
\hline NGB01234 & $8.14^{\mathrm{d}}$ & $6.50 \mathrm{~d}^{\mathrm{e}}$ & $1.78^{\mathrm{fg}}$ & $3.83^{\mathrm{abc}}$ & $1.02^{\mathrm{cd}}$ & $0.38^{\text {cde }}$ & $5.49^{\mathrm{ab}}$ & $1.79^{\mathrm{abcd}}$ \\
\hline NGB01613 & $14.14^{\mathrm{bc}}$ & $9.34 d^{c}$ & $3.18^{\text {cde }}$ & $2.03^{\mathrm{efg}}$ & $0.88^{\mathrm{cd}}$ & $0.33^{\mathrm{de}}$ & $1.94^{\mathrm{de}}$ & $1.35^{\text {cde }}$ \\
\hline NGB01662 & $12.33^{b c d}$ & $6.59 \mathrm{~d}^{\mathrm{e}}$ & $2.23^{\mathrm{efg}}$ & $2.92^{\text {bcde }}$ & $0.75^{\mathrm{d}}$ & $0.46^{\mathrm{abcd}}$ & $1.84^{\mathrm{e}}$ & $1.76^{\mathrm{abcd}}$ \\
\hline
\end{tabular}

Mean with the same letter in the same column are not significantly at $\mathrm{p} \geq 0.05$ according to Duncan Multiple Range Test (DMRT) 
Table 6. Heritability and genotypic variance of Growth and yield traits of Amaranthus genotype

\begin{tabular}{lccc}
\hline Source of variation & Genotypic variance $\left(\mathrm{O}^{2} \mathrm{~g}\right)$ & Phenotypic variance $\left(\mathrm{O}^{2} \mathrm{p}\right)$ & Heritability $(\%)$ \\
\hline Plant height & 1322.040 & 1422.320 & 92.9 \\
Number of leaf & 2370.770 & 2637.910 & 89.9 \\
Stem length & 970.868 & 1016.970 & 95.5 \\
Stem girth & 0.169 & 0.184 & 91.8 \\
Leaf length & 31.879 & 35.064 & 90.9 \\
Leaf width & 7.048 & 7.649 & 92.1 \\
Number of inflorescent & 140.111 & 256.426 & 54.6 \\
Inflorescent length & 68.186 & 113.060 & 60.3 \\
Inflorescent width & 6.689 & 11.207 & 59.7 \\
Number of branches & 16.683 & 22.638 & 73.7 \\
Weight of fresh leaf & 0.854 & 1.809 & 89.4 \\
Weight of dry leaf & 0.085 & 0.181 & 47.2 \\
Wet yield & 10.749 & 22.776 & 47.2 \\
Weight of dry inflorescent & 2.081 & 4.409 & 47.2 \\
\hline
\end{tabular}

Table 7. Principal Components Analysis (PCA) of Growth and Yield Characters of Genotypes of Amaranthus spp.

\begin{tabular}{|c|c|c|c|c|c|c|c|c|c|c|c|c|c|c|}
\hline Characters & Prin. 1 & Prin. 2 & Prin. 3 & Prin. 4 & Prin. 5 & Prin. 6 & Prin. 7 & Prin. 8 & Prin. 9 & Prin. 10 & Prin. 11 & Prin. 12 & Prin. 13 & Prin. 14 \\
\hline PH & -0.2519 & 0.3319 & -0.1109 & -0.1746 & 0.4813 & -0.2049 & 0.2745 & -0.1748 & 0.043 & -0.1351 & -0.1294 & -0.0597 & 0.603 & -0.0037 \\
\hline NL & -0.0793 & 0.2423 & -0.0578 & 0.6169 & 0.2308 & 0.6235 & -0.0817 & -0.0711 & -0.1436 & 0.1706 & 0.1232 & .1214 & 0.1327 & 0.0072 \\
\hline SL & -0.2922 & 0.3213 & -0.1811 & -0.0807 & 0.4099 & -0.1621 & 0.0811 & -0.0191 & -0.0523 & 0.0349 & 0.1219 & 0.1493 & -0.7278 & -0.0012 \\
\hline SG & -0.1766 & 0.3794 & -0.2224 & 0.1167 & -0.1888 & -0.1767 & -0.7272 & 0.1763 & 0.1235 & -0.2963 & -0.1586 & -0.0441 & 0.0695 & -0.0063 \\
\hline LL & -0.1506 & 0.3805 & -0.0073 & 0.0298 & -0.5711 & 0.2148 & 0.4703 & -0.1988 & 0.0316 & -0.4241 & -0.0607 & -0.0051 & -0.1188 & -0.0039 \\
\hline LB & -0.2769 & 0.3092 & -0.0828 & -0.2519 & -0.3689 & -0.0291 & 0.0325 & 0.1959 & -0.0882 & 0.7038 & 0.2215 & -0.0612 & 0.1579 & 0.0095 \\
\hline NI & 0.0187 & 0.2209 & 0.5883 & -0.0789 & 0.08 & -0.0233 & -0.0095 & 0.4288 & -0.6027 & -0.2084 & 0.0034 & 0.0117 & 0.0266 & 0.0059 \\
\hline IL & 0.2836 & 0.2651 & 0.2814 & -0.0206 & -0.0707 & -0.1949 & -0.2414 & -0.7397 & -0.2199 & 0.2231 & -0.1318 & 0.0521 & -0.0534 & 0.0115 \\
\hline IB & 0.0173 & 0.2181 & 0.5409 & -0.2649 & 0.1395 & 0.3079 & -0.1209 & 0.0414 & 0.6692 & 0.029 & 0.0841 & 0.0005 & -0.0529 & -0.0106 \\
\hline NB & 0.1374 & 0.1679 & 0.2182 & 0.6181 & -0.0439 & -0.5166 & 0.273 & 0.2163 & 0.2964 & 0.1835 & -0.1041 & -0.0144 & -0.0217 & 0.0106 \\
\hline $\begin{array}{l}\text { Weight of } \\
\text { fresh leaf }\end{array}$ & 0.3964 & 0.1669 & -0.1371 & -0.0233 & -0.0132 & -0.1627 & -0.0099 & -0.0066 & 0.0341 & 185 & 0.8057 & 0.1976 & 15 & 0.1083 \\
\hline $\begin{array}{l}\text { Weight of } \\
\text { dry leaf }\end{array}$ & 0.3974 & 0.1883 & -0.1959 & -0.1415 & 0.0103 & 0.0813 & 0.0702 & 814 & 0.0107 & 0.0716 & -0.2432 & 0.3997 & 0.0239 & -0.6921 \\
\hline $\begin{array}{l}\text { Weight of } 0.384 \\
\text { fresh inflorescer }\end{array}$ & $\begin{array}{l}10.2196 \\
t\end{array}$ & -0.1719 & -0.0271 & 0.1214 & 0.1177 & 0.0467 & 0.0682 & -0.0428 & 0.0203 & 0.0147 & -0.8392 & -0.1314 & -0.1201 & \\
\hline $\begin{array}{l}\text { Weight of } \\
\text { dry inflorescent }\end{array}$ & 0.3895 & 0.1929 & -0.2071 & -0.1554 & 0.0372 & 0.1332 & 0.0747 & 0.1947 & 0.0163 & 0.0865 & -0.3626 & 0.218 & -0.0222 & 0.7029 \\
\hline Eigenvalue & 4.7269 & 2.9902 & 1.9577 & 1.3231 & 0.8779 & 0.5563 & 0.4175 & 0.3249 & 0.2511 & 0.2197 & 0.1618 & 0.1054 & 0.0837 & 0.0038 \\
\hline Proportion & 0.3376 & 0.2136 & 0.1398 & 0.0945 & 0.0627 & 0.0397 & 0.0298 & 0.0232 & 0.0179 & 0.0157 & 0.0116 & 0.0075 & 0.006 & 0.0003 \\
\hline
\end{tabular}

PH: Plant Height, NL: Number of leaves, SL: Stem Length, SG: Stem Girth, LL: Leaf Length, LB: Leaf Width, NI: Number of Inflorescent, IL: Inflorescent Length, IB: Inflorescent Width, NB: Number of Branches

Table 8. Correlation Coefficient among Characters in Genotype of Amaranthus spp.

\begin{tabular}{|c|c|c|c|c|c|c|c|c|c|c|c|c|c|c|c|}
\hline PH & $\mathrm{PH}$ & NL & SL & SG & LL & LB & NI & IL & IB & NB & $\begin{array}{l}\text { Weight of } \\
\text { fresh leaf } \\
\text { inflorescent }\end{array}$ & $\begin{array}{l}\text { Weight of } \\
\text { dry leaf } \\
\text { inflorescent }\end{array}$ & $\begin{array}{l}\text { Weight of } \\
\text { fresh dry } \\
\text { inflorescent }\end{array}$ & $\begin{array}{l}\text { Genotype } \\
\text { pe }\end{array}$ & Week \\
\hline$\overline{\mathrm{NL}}$ & $0.5331^{*}$ & & & & & & & & & & & & & & \\
\hline SL & $0.9746^{* *}$ & $0.5748^{*}$ & & & & & & & & & & & & & \\
\hline SG & $0.7485^{* *}$ & $0.5755^{*}$ & $0.7909^{* *}$ & & & & & & & & & & & & \\
\hline LL & 0.2207 & 0.2627 & 0.2362 & 0.4259 & & & & & & & & & & & \\
\hline LB & $0.5678^{*}$ & 0.2423 & $0.6090^{* *}$ & $0.6603^{* *}$ & $0.6210^{* *}$ & & & & & & & & & & \\
\hline NI & $0.6840^{* *}$ & 0.3955 & $0.6464^{* *}$ & 0.4721 & 0.163 & 0.3416 & & & & & & & & & \\
\hline IL & $0.6420^{* *}$ & 0.3929 & $0.5913^{*}$ & 0.4965 & 0.1064 & 0.1994 & $0.7503^{* *}$ & & & & & & & & \\
\hline IB & $0.7217^{* *}$ & 0.3809 & $0.6752^{* *}$ & 0.4808 & 0.1565 & 0.3681 & $0.8671^{* *}$ & $0.7537 * *$ & & & & & & & \\
\hline NB & $0.5567^{*}$ & $0.5712^{*}$ & $0.5391 *$ & 0.4837 & 0.1152 & 0.1129 & $0.6351 * *$ & $0.7012^{* *}$ & $0.5449^{*}$ & & & & & & \\
\hline $\begin{array}{l}\text { Weight of } \\
\text { fresh leaf }\end{array}$ & 0.3688 & 0.2085 & 0.3267 & 0.3000 & $0-0.0442$ & $0-0.0585$ & 0.3766 & $0.7165^{* *}$ & 0.3871 & $0.5296^{*}$ & & & & & \\
\hline $\begin{array}{l}\text { Weight of } \\
\text { dry leaf }\end{array}$ & 0.3889 & 0.2215 & 0.3443 & 0.3027 & $0-0.0254$ & $0-0.002$ & 0.3653 & $0.6918^{* *}$ & 0.4023 & 0.4548 & $0.8973 * *$ & & & & \\
\hline $\begin{array}{l}\text { Weight of } \\
\text { fresh inflorescent }\end{array}$ & 0.3957 & 0.3137 & 0.3535 & 0.3116 & $0-0.0224$ & $0-0.0342$ & 0.374 & $0.6964 * *$ & 0.4008 & 0.4927 & $0.8856^{* *}$ & $0.9210^{* *}$ & & & \\
\hline $\begin{array}{l}\text { Weight of } \\
\text { dry inflorescent }\end{array}$ & 0.3928 & 0.2279 & 0.348 & 0.3029 & $0-0.0229$ & 0.0035 & 0.3561 & $0.6765^{* *}$ & 0.4017 & 0.432 & $0.8722 * *$ & $0.9940 * *$ & $0.9321 * *$ & & \\
\hline Genotype & $0-0.1166$ & 0.1491 & -0.0882 & -0.0121 & -0.0595 & $0-0.1119$ & $0-0.0466$ & $0-0.0221$ & $0-0.0641$ & 0.0371 & $0-0.0475$ & 0.0058 & $0-0.0083$ & 0.0036 & \\
\hline Week & $0.8919^{* *}$ & $0.5257^{*}$ & $0.8741^{* *}$ & $0.6648^{* *}$ & 0.0642 & 0.3816 & $0.7279 * *$ & $0.7595 * *$ & $0.7530^{* *}$ & $0.6756^{* *}$ & $0.5175^{*}$ & $0.5271^{*}$ & $0.5097^{*}$ & $0.5208^{*}$ & 0 \\
\hline Replicate & $0-0.0033$ & $0-0.0035$ & $0-0.0023$ & $0-0.0207$ & $0-0.0155$ & $0-0.0032$ & 0.0548 & $0-0.0133$ & 0.0174 & $0-0.002$ & 0.00000 & 0.0000 & 0.00000 & 0.0000 & \\
\hline
\end{tabular}
Inflorescent, IL: Inflorescent Length, IB: Inflorescent Width, NB: Number of Branches

Principal Components Analysis (PCA) of Growth and Yield Characters of Genotypes of Amaranthus spp.

The result from Table 7 delineates the Amaranthus genotype into fourteen principal component axes; Prin.
$1,2,3,4,5,6,7,8,9,10,11,12,13$ and 14. Prin. 1 which constituted the highest accounted for 0.3376 of the total proportion with eigen value of 4.7269 , while Prin. 14 was the least with proportion of 0.0003 and eigenvalue of 0.0038 . Weight of dry leaf from Prin. 1 
had the highest eigen vector of 0.3973 while Number of leaves was the least with $(-0.0793)$. Also Prin. 2 produced the highest eigen vector for Leaf length at 0.3805 while weight of fresh leaf biomass had the least at 0.1669 . Prin. 3 produced the highest eigen vector at 0.5883 for number of inflorescent while Leaf length produced the least at (0.0073). Prin. 4 produced the highest eigen vector at 0.6181 for Number of branches while Inflorescent length had the least at $(-0.0206)$. Prin. 5 produced the highest eigen vector at 0.4813 for Plant height while weight of fresh leaf biomass had the least at $(-0.0132)$. Prin. 6 produced the highest eigen vector at 0.6235 for Number of leaves while Number of Inflorescent had the least at (0.0233). Prin. 7 produced the highest eigen vector at 0.4703 for leaf length while Weight of fresh leaf biomass had the least at $(-0.0095)$. Prin. 8 produced the highest eigen vector at 0.4288 for number of inflorescent while Weight of fresh leaf had the least at $(-0.0066)$. Prin. 9 produced the highest eigen vector at 0.6692 for inflorescent width while Weight of fresh inflorescent biomass had the least at $(-0.0428)$. Prin. 10 produced the highest eigen vector at 0.7038 for Leaf width while Plant height has the least at $(-0.1351)$. Prin. 11 produced the highest eigen vector at 0.8057 for Weight of fresh leaf biomass while leaf length has the least at $(-0.0607)$. Prin. 12 produced the highest eigen vector at 0.3997 for Weight of dry leaf biomass while leaf length has the least at (0.0051 ). Prin. 13 produced the highest eigen vector at 0.6030 for plant height while number of Branches has the least at $(-0.0217)$. Prin. 14 produced the highest eigen vector at 0.7029 for Weight of dry inflorescent biomass while Stem length has the least at (-0.0012).

\section{Correlation Co-efficient among Characters in Genotype of Amaranthus spp.}

The correlation result is shown in Table 8. The plant height had a positive significant correlation with Number of leaf $(r=0.5331)$, Leaf width $(r=0.5678)$, Number of branches $(\mathrm{r}=0.5567)$ and a strong positive correlation with Stem length $(r=0.9746)$, Stem girth $(r=0.7485)$, Number of inflorescent $(\mathrm{r}=0.6840)$, Inflorescent length (0.6420), Inflorescent width $(\mathrm{r}=0.7217)$ and Week $(\mathrm{r}=$ $0.8919)$. Number of leaf had a positive correlation with Stem length $(\mathrm{r}=0.5748)$, Stem girth $(\mathrm{r}=0.5755)$, Number of branches $(r=0.5712)$ and Week $(r=0.5257)$. In addition, Stem length showed a strong positive correlation with Stem girth $(\mathrm{r}=0.7909)$, Leaf width $(\mathrm{r}=$ 0.6090), Number of inflorescent $(\mathrm{r}=0.6464)$, Inflorescent width $(\mathrm{r}=0.6752)$ and Week $(\mathrm{r}=0.8741)$ and a positive correlation with Inflorescent length (0.5913) and Number of branches ( $\mathrm{r}=0.5391)$. Moreover, Stem girth had a strong positive correlation with Leaf width $(r=0.6603)$ and Week $(r=0.6648)$. Leaf length had a strong positive correlation with Leaf width $(r=0.6210)$. Number of inflorescent had a strong positive correlation with Inflorescent length $(r=0.7503)$, Inflorescent width $(r=0.8671)$, Number of branches $(r=$
$0.6351)$ and Week $(r=0.7279)$. Inflorescent length had a strong positive correlation with Inflorescent width $(\mathrm{r}=$ 0.7537), Number of branches $(r=0.7012)$, Weight of fresh leaf $(r=0.7165)$, Weight of dry leaf $(r=0.6918)$, Weight of fresh inflorescent $(\mathrm{r}=0.6964)$, Weight of dry inflorescent $(r=0.6765)$ and Week $(r=0.7595)$. Inflorescent width had a strong positive correlation with Week $(r=0.6756)$ and a positive correlation with Number of branches $(r=0.5449)$. Number of branches had a strong positive correlation with Week $(r=0.6756)$ and a positive correlation with Weight of fresh leaf $(r=0.5296)$. Weight of fresh leaf had a strong positive correlation with Weight of dry leaf $(r=0.8973)$, Weight of fresh inflorescent $(\mathrm{r}=0.8856)$, Weight of dry inflorescent $(\mathrm{r}=0.8722)$ and a positive correlation with Week $(r=0.5175)$. Weight of dry leaf had a strong positive correlation with Weight of fresh inflorescent $(r=0.9210)$, Weight of dry inflorescent $(r=$ $0.9940)$ and a positive correlation with Week $(r=0.5271)$. Weight of fresh inflorescent had a strong positive correlation with Weight of dry inflorescent $(r=0.9321)$ and a positive correlation with Week $(r=0.5097)$. While Weight of dry inflorescent had a positive correlation with Week $(\mathrm{r}=0.5208)$.

\section{Dendogram Showing the Relationship of Yield Characters Among the Amaranthus Genotypes}

The dendogram showing the relationship of Yield Characters among the Amaranthus Genotypes is shown in Fig. 1. There are two major clusters sub-divided into four (4) groups. Genotype NG/SA/DEC/07/0423 and NGBO1271 is closely related but different from genotype NGB01613 while genotype NGB01667 and NGB01283 is similar than genotype NGB01276. Also, genotype NGB01259 and NGB01644 are closely related compared to genotype NGB01662. Again, genotype NG/AA/03/11/010 and NG/AA/MAY/09/027 are closely related than genotype NGB01234 while genotype NG/AO/11/08/042 and NG/SA/DEC/07/0412 are similar as also observed in genotype NG/AO/11/08/039 and NGB01601.

\section{Dendogram Showing the Relationship of Growth Characters Among the Amaranthus Genotypes}

The relationship of Growth Characters among the Amaranthus Genotypes is depicted in the dendogram as shown in Fig. 2. There are three major clusters subdivided into five (5) groups. Genotype NG/AO/11/08/042 and NG/SA/DEC/07/0412 are similar compared to genotype NG/AA/MAY/09/027 while Genotype NGB01667 and NGB01271 are similar but different from NG/SA/DEC/07/0423 and NGB01283. Also, genotype NGB01276 and NGB01613 are closely related compared to genotype NGB01601. Again, Genotype NG/AO/11/08/039 and NGB01234 are similar but different from genotype NGB01259 and NGB01644 while Genotype NG/AA/03/11/010 and NGB01662 are similar as seen in Fig. 2. 


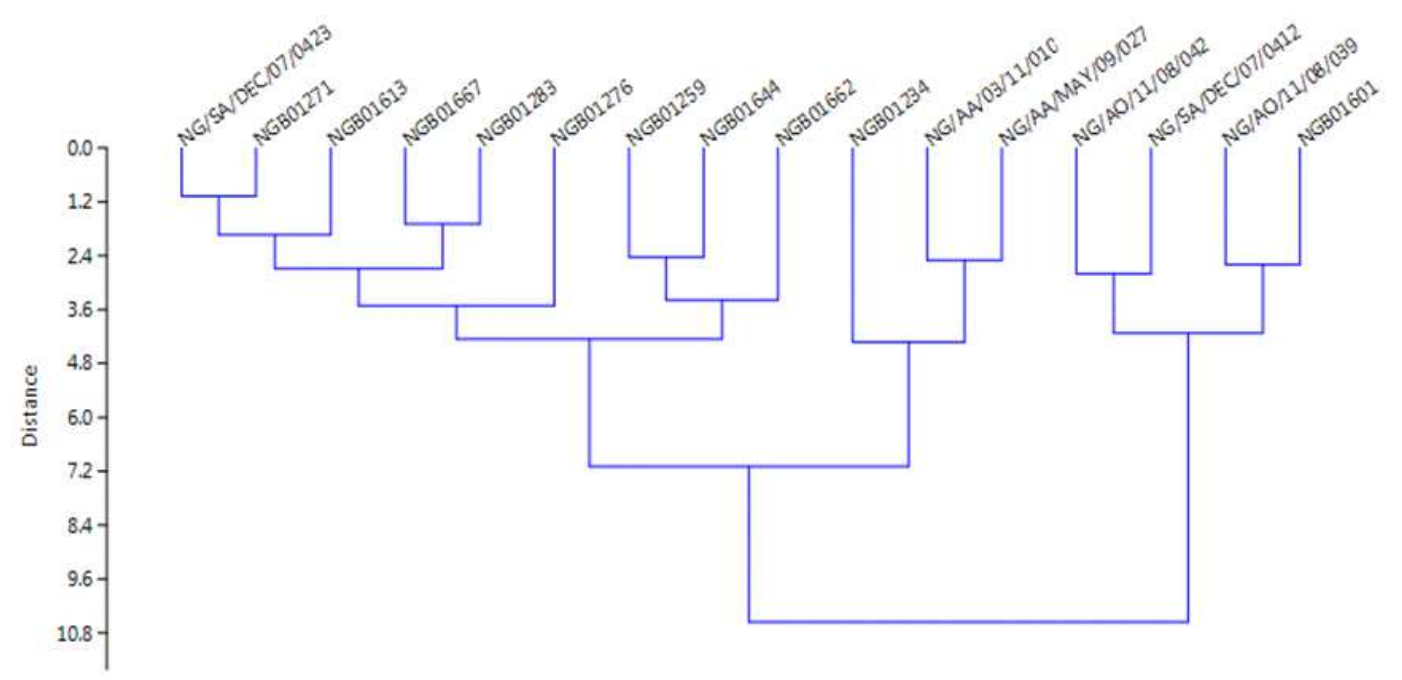

Fig. 1. Showing the relationship of yield characters among the Amaranthus genotypes

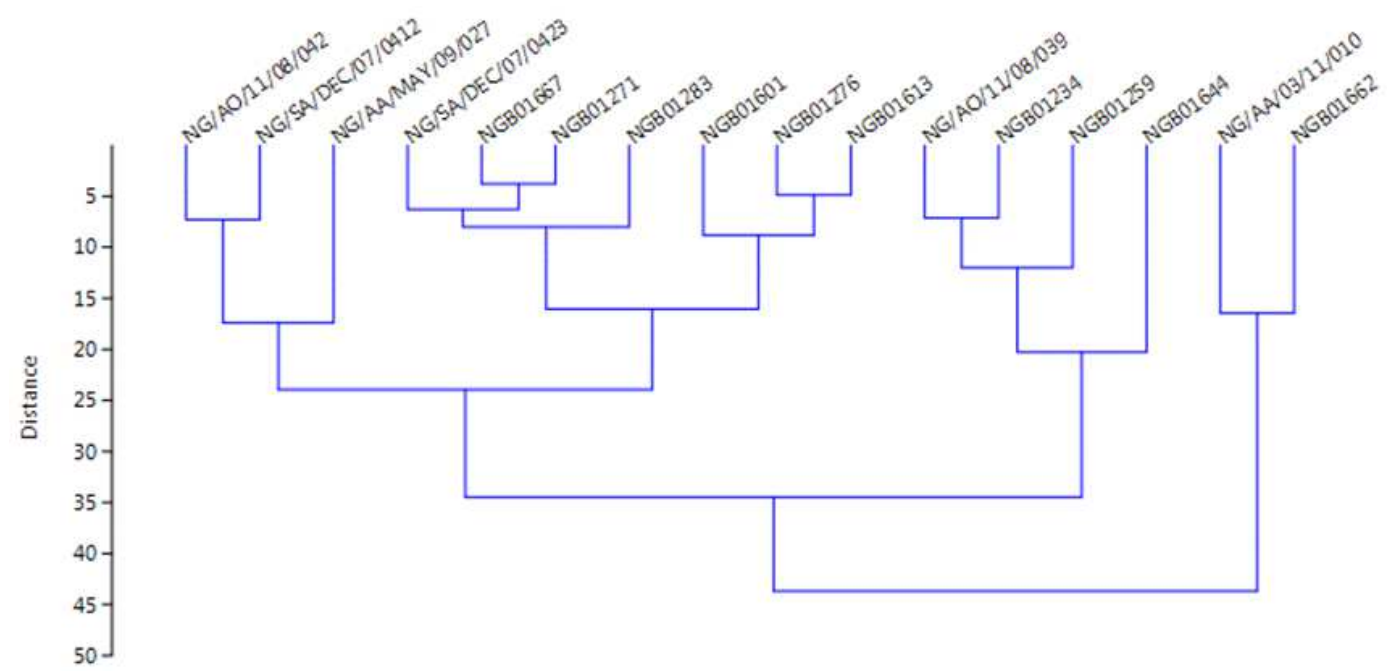

Fig. 2. Showing the relationship of growth characters among the Amaranthus Genotypes

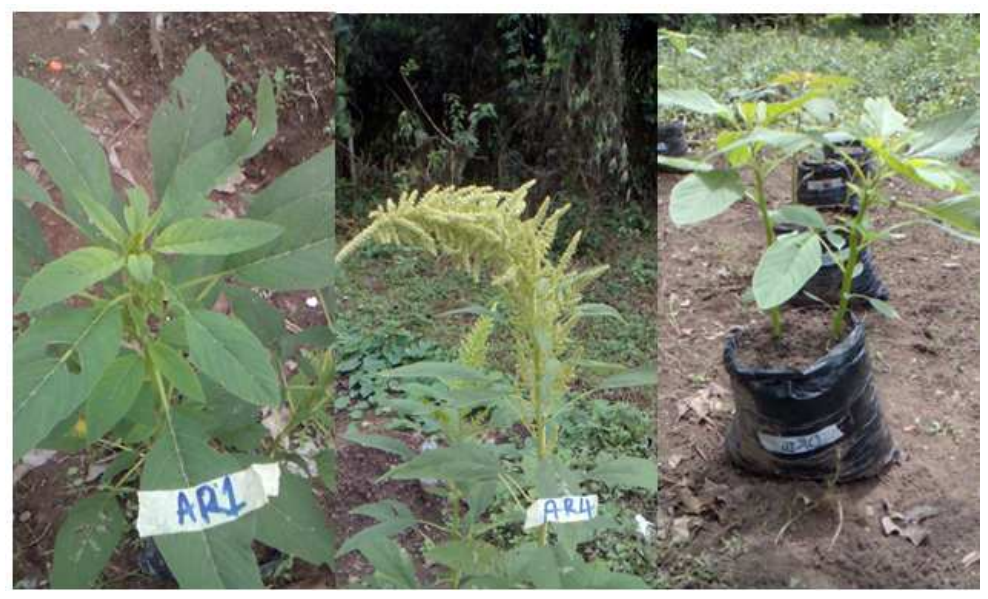

Fig. 3. NG/AA/MAY/09/027 genotype showing the best growth character 


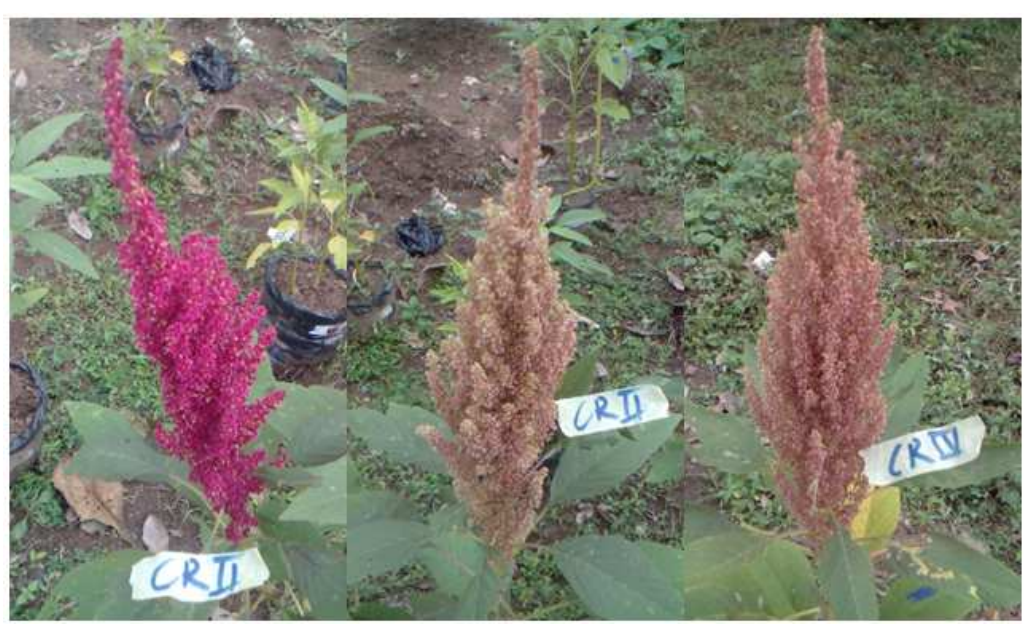

Fig. 4. NG/AO/11/08/042 genotype showing the best yield attribute

\section{Discussion}

The findings from this study showed that there are variations in the performance of growth and yield characters studied among the Amaranthus genotypes. This is in accordance with the reports of Nwangburuka et al. (2012) and Olawuyi et al. (2014) who considered genetic variability as essential in crop breeding. The genotypic effect also had significant expression on the traits evaluated in Amaranthus. Variability in performance of genotypes also affected the growth performance of Amaranthus. The variations shown by the characters were due to high genetic diversity, differences of growing type and differences on the type of adaptation (Kulakow, 1987; Mujica and Jacobsen, 2003).

The best performance of growth and yield characters exhibited by NG/AA/MAY/09/027, NG/AO/11/08/039 and NG/AO/11/08/042 genotypes (Fig. 3 and Fig. 4) could be due to genetic variation of these genotypes. Selections based on this characters and the genetic diversity inherent in the plants could thus improve productivity considerably. These performances shown by Amaranthus also suggest hybridization breeding procedure for crop improvement with desired traits in the parents line.

The findings from correlation coefficient shows that the characters were mostly positively related as similarly observed by Olawuyi et al. (2012). The correlation between the characters implies that selection based on plant height will favour all growth and yield characters. This will enhance the rate of productivity and yield.

Prin 1 accounted for the highest variation as previously observed by Olowe et al. (2013) and Olawuyi et al. (2015). The results of the Principal components analysis reveals the pattern of variation among the characters studied and the characters that accounted most for variation within a group of entries (Ogunbodede and Omueti, 1997; Aremu et al., 2007). It implies that the Principal Component Analysis (PCA) can be quantified from the contribution of the different variable to each principal component as revealed by the eigen vector (Lezzoni and Pritts, 1991).

This phenotypic expressions might be due to environmental influences; exacerbating this problem is the presence of considerable morphological variation within cultivated populations (Sauer, 1967; Espitia, 1992). This shows that their genotypes and species genetic make-up played a huge role in the phenotypes expressed in this studies.

The phenotypic variance of both growth and yield characters were higher than the genotypic variance in all the characters studied. Heritability of growth traits were higher than yield traits in Amaranthus genotypes. This shows that the proportion of genotypic effect to phenotypic effect was higher at growth than maturity. This conforms to the report of Palaniappan et al. (1999) who observed an improvement in general crop performance. This supported the findings of Chadha and Paul, 1984; Gautam and Srinivas, 1992; Prasad et al., 2004; Singh and Kumar, 2005; Babu and Patil, 2005 who reported high heritability and genetic advance for yield characters for Solanum melongena.

\section{Conclusion and Recommendation}

The variations in the genotypes could be sufficient basis for crop improvement. NG/AA/MAY/09/027, $\mathrm{NG} / \mathrm{AO} / 11 / 08 / 039$ and $\mathrm{NG} / \mathrm{AO} / 11 / 08 / 042$ are promising genotypes that could be selected and explored for future breeding in improvement of Amaranthus vegetable. This will further enhance proper documentation and conservation of Amaranthus germplasm. 


\section{Acknowledgement}

Special thanks to Department of Botany, University of Ibadan, Oyo state, Nigeria for making their facilities accessible for the research work. Also, unreserved appreciation goes to National Centre for Genetic Resources and Biotechnology (NACGRAB), Moor plantation, Ibadan, Nigeria for providing the seeds.

\section{Funding Information}

This work was supported and funded by both authors.

\section{Author's Contributions}

This work was carried out in collaboration among the authors. Both authors participated actively and synergistically in all works, analysis and the entire process of the writing, editing and approval of the manuscript.

\section{Ethics}

The authors declares that there is no conflict of interest and this manuscript conforms to the ethical standards specified by the American Journal of Agricultural and Biological Sciences.

\section{References}

Amanambu, C.A. and C.N. Egbinola, 2013. Climate variation assessment based on rainfall and temperature in Ibadan, South-Western. Nig. J. Environ. Earth Sci., 3: 2224-3216.

Angel, H.O. and B.R. Paulina, 2011. Amaranth: A pseudo-cereal with nutraceutical properties. Curr. Nutriti. Food Sci., 7: 1-9.

Aremu, C.O., M.A. Adebayo, M. Oyegunle and J.O. Ariyo, 2007. The relative discriminatory abilities measuring Genotype by environment interaction in soybean (Glycine max). Agric. J., 2: 210-215

Babu, S.R. and R.V. Patil, 2005. Genetic variability and correlation studies inegg plant (Solanummelongena L.). Madras J. Aric. Res., 95: 18-23.

Brenner, D.M., D.D. Baltensperger, P.A. Kulakow, J.W. Lehmann and R.L. Myers et al., 2010. Genetic Resources and Breeding of Amaranthus. In: Plant Breeding Reviews, Jules J. (Ed.), John Wiley and Sons, Hoboken, ISBN-10: 0470650087 , pp: 227-285.

Bressani, R., A. Sanchez-Marroquin and E. Morales, 1992. Chemical composition of grain amaranth cultivars and effects of processing on their nutritional quality. Food Rev. Int., 8: 23-49.

Buragohain, J., V.B. Singh, B.C. Deka, A.K. Jha and $\mathrm{K}$. Wanshnong et al., 2013. Collection and evaluation of some underutilized leafy vegetables of Meghalaya Indian. J. Hill Farming, 26: 111-115.
Chadha, M.L. and B. Paul, 1984. Genetic variability and correlation studies in eggplant (Solanummelongena L.). Indian J. Hort., 41: 101-107.

Espitia, E., 1992. Amaranth germplasm development and agronomic studies in Mexico. Food Rev. Int., 8: 71-86.

Gautam, B. and T. Srinivas, 1992. Study on heritability, genetic advance and characters association in brinjal (Solanummelongena L.). SouthIndian Hort, 40: 316-318.

Holm, L., J. Doll, E. Holm, J. Pancho and J. Herberger, 1997. World weeds: Natural histories and distribution. 1st Edn., John Wiley and Sons, Toronto.

Kulakow, P.A., 1987. Genetics of grain amaranths. J. Hered. 78: 293-297.

Lezzoni, A.F. and M.P. Pritts, 1991. Application of principal component analysis to horticultural research. Hort. Sci., 26: 334-338.

Mujica, A. and S.E. Jacobsen, 2003. The genetic resources of Andean grain amaranths (Amaranthus caudatus L., A. cruentus L. and A. hypochondriacus L.) in America. Plant Genetic Resources Newsletter, 133: 41-44.

Nwangburuka, C.C., O.A. Denton, O.B. Kehinde, D.K. Ojo and A.R. Popoola, 2012. Genetic variability and heritability in cultivated okra (Abelmoschusesculentus[L.] moench). Spanish J. Agric. Res., 10: 123-129.

Ogunbodede, B.A. and O. Omueti, 1997. Regional research project on maize and cassava report on research activities on maize breeding and utilization. Sponsored Eleven Coastal West African Countries.

Olawuyi, O.J., D.T. Ezekiel-Adewoyin, A.C. Odebode, D.A. Aina and G.E. Esenbamen, 2012. Effect of arbuscularmycorrhizal fungi (Glomusclarum) and organomineral fertilizer on growth and yield performance of Okra (Abelmoschusesculentus). African J. Plant Sci., 6: 84-88.

Olawuyi, O.J., S.G. Jonathan, F.E. Babatunde, B.J. Babalola and O.S. Yaya et al., 2014. Accession $\mathrm{x}$ treatment interaction, variability and correlation studies of pepper (Capsicum spp.) under the influence of ArbuscularMycorrhiza Fungus (Glomusclarum) and Cow Dung. Am. J. Plant Sci., 5: 683-690.

Olawuyi, O.J., O.B. Bello, C.V. Ntube and A.O. Akanmu, 2015. Progress from selection of some maize cultivars' response to drought in thederived savanna of Nigeria. AGRIVITA J. Agric. Sci., 37: 8-17.

Olowe, O.M., A.C. Odebode, O.J. Olawuyi and A.O. Akanmu, 2013. Correlation, principal component analysis and tolerance of maizegenotypes to drought and diseases in relation to growth traits. Am. Eurasian J. Agric. Environ. Sci., 13: 1554-1561.

Palaniappan, S.P., A. Jeyabal and S. Chelliah, 1999. Evaluation of integrated nutrient management in summer sesame (Sesamum indicum L.). Sesame Saffl. Newslett. 
Prakash, D. and M. Pal, 1991. Nutritional and anti nutritional composition of vegetable and grain amaranth leaves. J. Sci. Food Agric., 57: 573-583.

Prasad, M., N. Mehta, S.N. Diokshit and S.S. Nishal, 2004. Genetic variability, genetic advance and heritability in brinjal (Solanummelongena L.). Orissa J. Hort., 32: 26-29.

Sauer, J.D., 1967. The grain amaranths and their relatives: A revised taxonomic and geographic survey. Annals Missouri Botanical Garden, 54: 103-137.
Singh, O. and J. Kumar, 2005. Variability, heritability and genetic advance in brinjal. Indian J. Hort., 62: 265-267.

Steckel, L.E., 2007. The dioecious Amaranthus spp.: Here to stay. Weed Technol., 21: 567-570.

Tucker, J.B., 1986. Amaranth: The once and future crop. Bioscience, 36: 9-13. 